\title{
Depth-resolved mapping of muscular bundles in myocardium pulmonary junction using optical coherence tomography
}

Zhifang Li

Qinggong Tang Timm Dickfeld

Yu Chen 


\title{
Depth-resolved mapping of muscular bundles in myocardium pulmonary junction using optical coherence tomography
}

\author{
Zhifang Li, ${ }^{a}$ Qinggong Tang, ${ }^{b}$ Timm Dickfeld, ${ }^{c}$ and Yu Chen ${ }^{a, b, *}$ \\ ${ }^{a}$ Ministry of Education, Fujian Normal University, College of Photonic and Electronic Engineering, Fujian Provincial Engineering Technology \\ Research Center of Photoelectric Sensing Application, Key Laboratory of Optoelectronic Science and Technology for Medicine, \\ Fujian Provincial Key Laboratory of Photonics Technology, Fuzhou, China \\ bUniversity of Maryland, Fischell Department of Bioengineering, College Park, Maryland, United States \\ 'University of Maryland, School of Medicine, Baltimore, Maryland, United States
}

\begin{abstract}
Atrial fibrillation (AF) is the most common cardiac arrhythmia and has high patient morbidity. One of the root causes of $A F$ is initiating triggers from atrial myocardium extending into the pulmonary veins. Visualizing the muscular bundles of myocardial extension is essential to guide the catheter radio-frequency ablation and confirm the curative tissue necrosis thereafter. We applied optical coherence tomography (OCT) for direct visualization of cardial muscle extension in myocardium pulmonary junction. Two perspectives (cross-sectional and en face images) are presented for imaging myocardial extensions. The results demonstrated that cross-sectional images can quickly locate the myocardium pulmonary junction. And en face images provide depth-resolved arrangement information of muscular bundles in the myocardium pulmonary junction. The results indicated that OCT could potentially be used to guide catheter radio-frequency ablation for treatment of AF. ๑ 2018 Society of PhotoOptical Instrumentation Engineers (SPIE) [DOI: 10.1117/1.JBO.23.7.076004]
\end{abstract}

Keywords: optical coherence tomography; muscular bundle; radius.

Paper 180082R received Feb. 6, 2018; accepted for publication Jun. 21, 2018; published online Jul. 6, 2018.

\section{Introduction}

Atrial fibrillation (AF) is the most common cardiac arrhythmia, particularly in the older population, and is associated with a high patient morbidity and health care costs. ${ }^{1}$ One of the root causes of $\mathrm{AF}$ is initiating triggers from atrial myocardium extending into the pulmonary veins (PV). The histological studies have shown that most of the focal triggers are located in the extensions of the left atrial myocardium (myocardial sleeves) into the proximal PVs. ${ }^{2}$ Currently, the locations of the myocardial sleeves at the PV ostia are identified indirectly using local electrical signals, and then radio-frequency ablating (RFA) is applied at the corresponding recording electrodes in the PVs. ${ }^{3}$ However, the recurrence rates of $50 \%$ or more are reported in longer-term follow-up evaluations as the muscular bundles progressively reconnect with the rest of the left atrial myocardium. Thus, there must be a thorough understanding of the anatomic structures in the myocardium pulmonary junction to carry out RFA.

Optical coherence tomography (OCT) and polarization-sensitive OCT have been applied for imaging myofiber arrangement in heart muscles. ${ }^{4-9}$ Since the orientation of myofiber tracts plays a central role in the function of the heart, a number of approaches have been reported to quantify the average fiber direction, such as ones based on the fast Fourier transform (FFT) 5 and edge detection. ${ }^{4,6-9}$ Recently, a weight orientation vector summation algorithm was presented for estimating fiber orientation at each pixel within a grayscale image to improve the accuracy of fiber orientation, assuming myofiber fills the entire image. ${ }^{10-12}$

Since muscular bundles are covered by fibrous tissues in myocardium pulmonary junction, in this study, we pay more attention to the extension of muscular bundles rather than the myofiber orientation. The goal of this study is using highresolution OCT image to understand the extension of muscular bundles in myocardium pulmonary junction. In this work, crosssectional and en face OCT images are used for locating and quantifying the extension of muscular bundles in myocardium pulmonary junction, respectively.

\section{Materials and Methods}

\subsection{Optical Coherence Tomography Image Acquisition}

Sheep hearts and adjacent lungs were procured from freshly slaughtered animals. The left and right PVs were opened with a longitudinal incision to visually identify the transition point of the myocardium to PV. We recruited eight samples in total from two different animals. Sheep myocardial sleeves were then imaged ex vivo by a high-speed, high-resolution swept-source OCT (SS-OCT) system. The details of the SSOCT system have been previously described. ${ }^{13-17}$ Briefly, the light source was a swept-source laser with 100-nm bandwidth centered at $1310 \mathrm{~nm}$, which yielded an axial resolution of $\sim 10 \mu \mathrm{m}$ in tissue. The lateral resolution was $\sim 15 \mu \mathrm{m}$. 


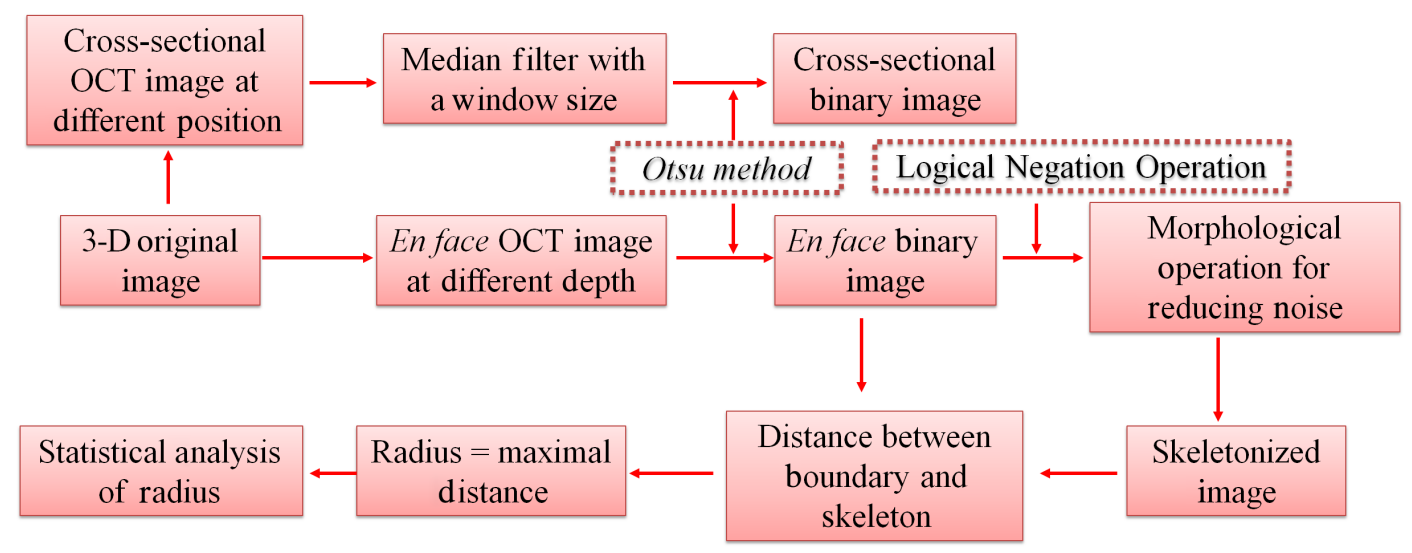

Fig. 1 Flowchart of extracting quantitative information of muscular bundle.

The imaging acquisition rate was $16 \mathrm{kHz}$ (30 frames per second).

\subsection{Cross-Sectional OCT Image Processing}

Cross-sectional OCT images $[\sim 8.0 \mathrm{~mm}(z) \times 4.2 \mathrm{~mm}(x)]$ of the extended muscular bundles were first filtered by a twodimensional (2-D) median filter with a window size of $5 \times 5$ to reduce the OCT speckle noise and then segmented by the Otsu's threshold for locating the muscular bundles in myocardium pulmonary junction, as shown in Fig. 1.

\subsection{Algorithm for Quantification of Muscular Bundles in En Face OCT Images}

The en face OCT images of muscular bundles in regions of interest (ROIs) were chosen for quantitative analysis. Figure 1 shows the overall flowchart of extracting depth-resolved quantitative information of muscular bundles. We used Otsu's threshold ${ }^{18}$ to generate the binary images and segment the muscular bundles, and then the negation operation was performed on binary images to visualize the muscular bundles. The binary image of muscular bundles was filtered by morphological open-close operation for reducing noise and keeping the shape ${ }^{19}$ The muscular bundles in the image were then shrunk to a center line based on the thinning algorithm, ${ }^{20}$ and finally, the processed image became a skeletonized image.

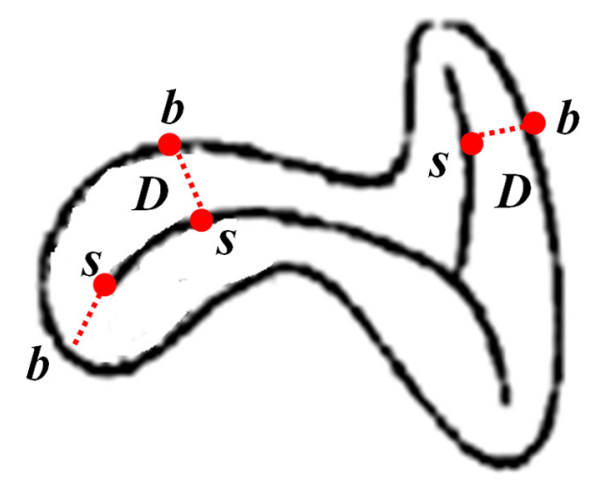

Fig. 2 The distance between the boundary and skeleton. " $b$ " is in the boundary and " $s$ " is in the skeleton.
The size of the muscular bundle can be quantified by the parameter of the radius. The local distance for every pixel $(s)$ on the skeleton was defined to be the minimal distance between the skeleton and pixel $b$ on the boundary set $(B)$ in the binary image as shown in Fig. 2

$D(b)=\min [\underset{\forall b \in B}{\operatorname{dist}(s, b)] .}$

The local radius was defined to be the maximal distance $D$

$R=\max (D)$.

Thus, we could obtain the spatial-resolved radius of muscular bundles at the different depths, and statistical analysis of the spatial-resolved radius was used for quantifying the feature of the muscular bundles.

\subsection{Statistical Analysis}

The radius is used to indicate the morphology of muscular bundles. Experimental data are shown as mean and standard deviation $(\mathrm{X} \pm \mathrm{SD})$. A two-sample $t$-test with unequal means was completed to determine whether the difference is significant in the statistical parameters between any two sample groups. Differences were considered to be statistically significant when $p<0.05$.

\section{Results}

\subsection{Distinguishing the Extensions of Myocardium from Cross-Sectional OCT Image}

The transition of atrial myocardium to PV in myocardium pulmonary junction can be distinguished by the color change from brown to white in the inset of Fig. 3. Figures 3(a)-3(c) are the cross-sectional OCT images filtered by a 2-D median filter. The segmented OCT images by the Otsu's threshold are shown in Figs. 3(d)-3(f), respectively. Figure 3(a) shows fibrous tissue from $\mathrm{PV}$, and the corresponding binary image shown in Fig. 3(d) indicates that PV has a homogeneous tissue structure. Figure 3(c) demonstrates that the myocardium exhibits a distinctive large dark structure. The wall of the myocardium contains a thin endothelium, and the myocardium in 

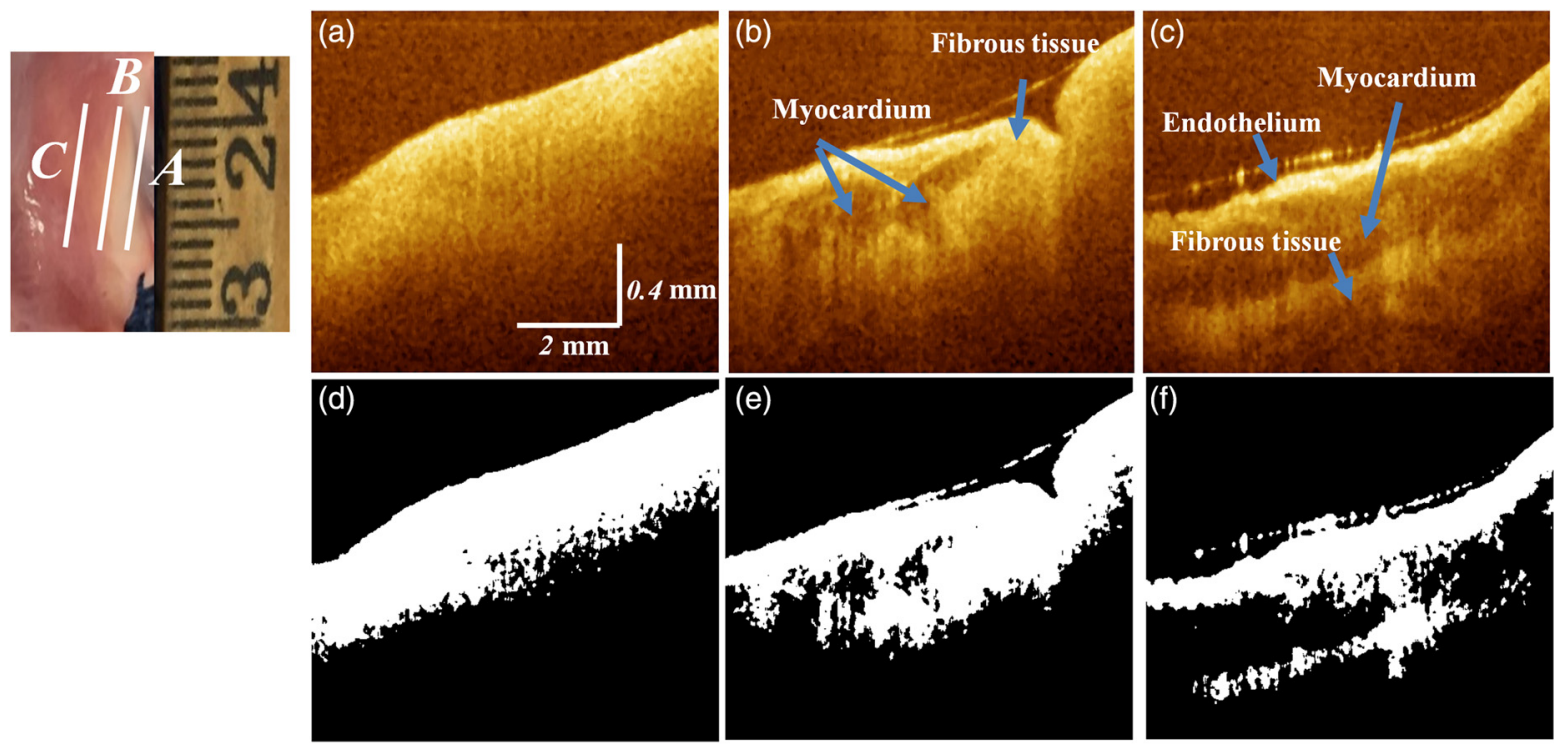

(g)

(h) Myocardium

(i)

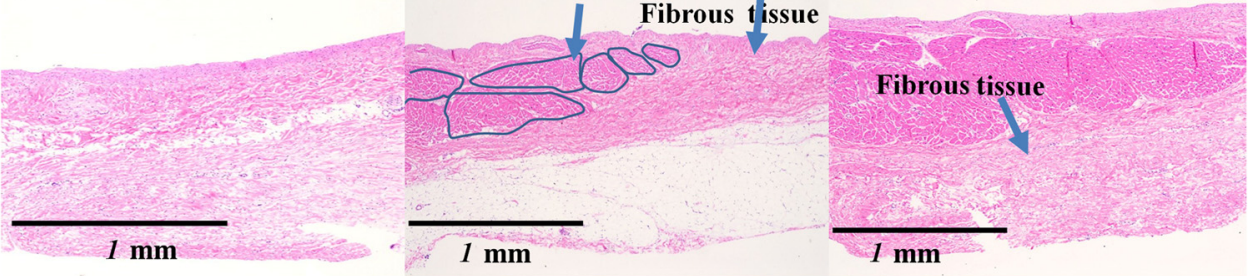

Fig. 3 Cross-sectional OCT images of (a) PV, (b) combined myocardium with PV, and (c) myocardium in myocardium pulmonary junction. The corresponding imaging areas were indicated in the inset labeled with $A, B$ and $C$ in picture. (d)-(f) are the corresponding binary images of (a)-(c), respectively.(g)-(i) are the corresponding histology image.

myocardium pulmonary junction is made up of smooth muscle and a thick outer fibrous tissue as indicated in Fig. 3(c). The muscular bundles show lower light scattering than that of PV, which is consistent with the findings of scattering coefficients of endocardium ${ }^{21}$ and vein. ${ }^{22}$ Figure 3(f) demonstrates that the endothelium and the outer fibrous tissue are separated by the myocardium in the myocardium PV. Figures 3(b) and 3(e) are the areas of combined myocardium with PV, and it can be observed that the extension of muscular bundle is segmented by fibrous tissue as shown in Fig. 3(h). The muscular bundle exhibits a distinctive large dark structure with lower light scattering as compared to adjacent fibrous tissue from PV that has higher light scattering and more homogeneous tissue structure. Thus, based on the scattering features of the myocardium and fibrous tissue, the cross-sectional binary images could estimate the extensions of muscular bundles in the PV.

\subsection{Morphology of Muscular Bundles of Myocardium Pulmonary Junction at Different Depths}

To obtain depth-resolved structure of the junctions, threedimensional OCT data were flattened and required to generate en face images $[\sim 5.4 \mathrm{~mm}(y) \times 2.7 \mathrm{~mm}(x)]$ at different depths. Figures 4(a)-4(c) demonstrate that the myocardium content gradually decreases, while the fibrous tissues become more prominent in PV regions, from left to right. They also show various morphologies of muscular bundles at different depths of
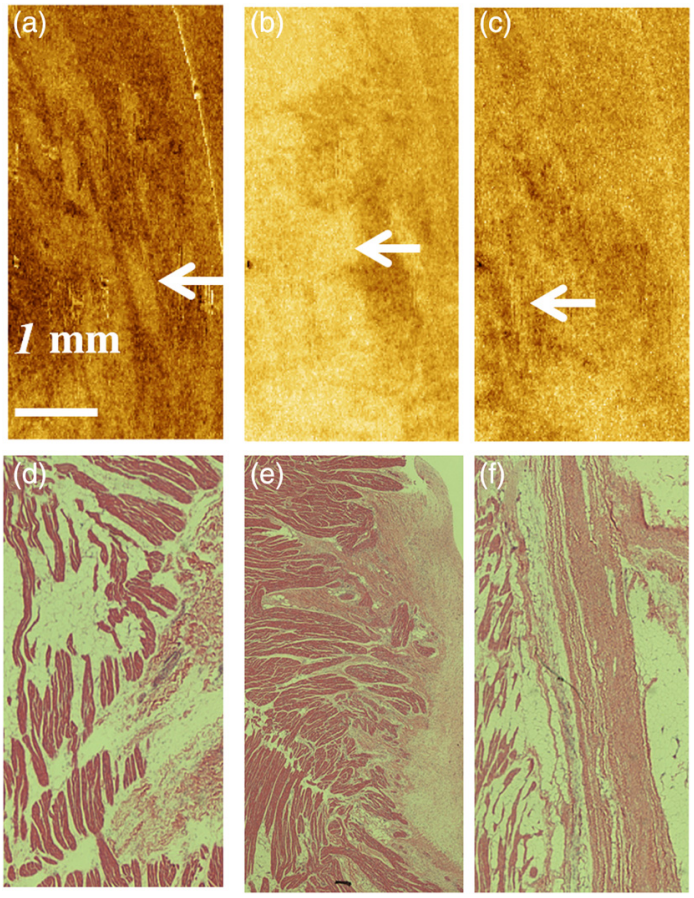

Fig. 4 Typical en face OCT images of myocardium pulmonary junction at different depths of (a) 40, (b) 340 , and (c) $560 \mu \mathrm{m}$. (d)-(f) are the corresponding histological sections. White arrows denote the regions of muscular bundles. From left to right, the myocardium content gradually decreases, while the fibrous tissues become more prominent in PV regions. 
(a)

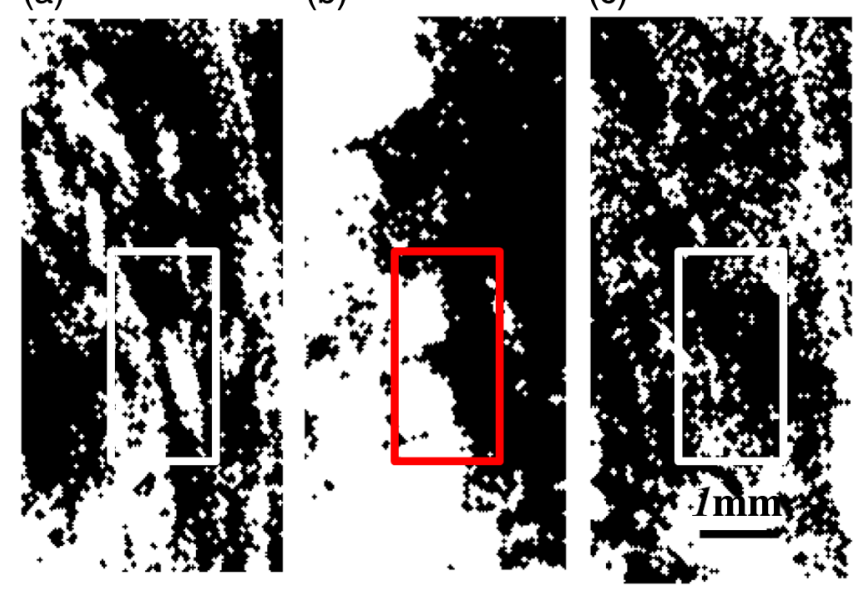

Fig. 5 Binary images of en face OCT image at different depths as shown in Figs. 4(a)-4(c), respectively. The rectangular boxes denote ROIs, which are used for estimation of radius. myocardium pulmonary junction from the en face OCT images. Since the wall of the myocardium contains a thin endothelium at the superficial layer, the muscular bundles were separated by the endothelium from the histological viewpoint as shown in Fig. 4(d). With increasing depth in the range from $\sim 80$ to $\sim 420 \mu \mathrm{m}$, the muscular bundles become much tighter with smaller separations between them [Fig. 4(e)]. In the en face OCT image as shown in Fig. 4(a), the region of the myocardium could be seen, while the muscular bundles become more blurry as the depth increases [Fig. 4(b)]. For deeper tissue in the range from 420 to $820 \mu \mathrm{m}$, the muscular bundles could be detected again as shown in Fig. 4(c), since the connective tissue deposition increases between muscular bundles at the deeper regions [Fig. 4(f)]. These phenomena are consistent with median radius distributions in Fig. 6(a), which demonstrated that there are obviously three layers. Thus, the myocardium pulmonary junction can be typically divided into three distinct layers based on the structure changes of muscular bundles at different depths.

(a)
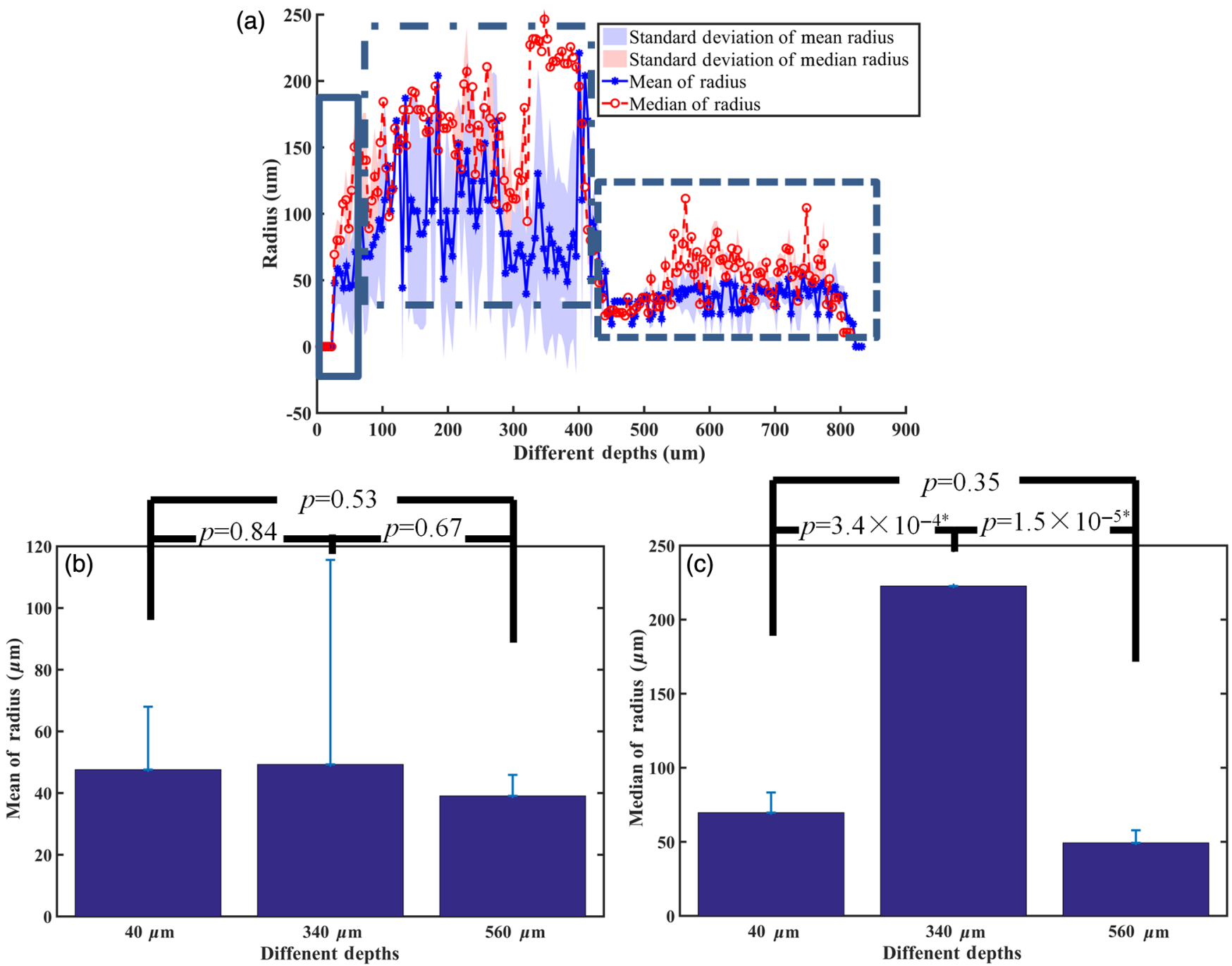

Fig. 6 (a) Mean and median of radius in the ROls at different depths of myocardium pulmonary junction. The dot rectangular boxes denote the different layers. (b) Mean and (c) median are selected from some typical depths. Data are expressed as mean \pm standard deviation $(n=8)$. "*” denote statistical significant values $(p<0.05)$. 


\subsection{Quantification of Muscular Bundles at the Different Depths}

To quantify the muscular bundles at different depths in myocardium pulmonary junction region, the Otsu's threshold was employed to generate the binary image and segment the muscular bundles as shown in Figs. 5(a)-5(c). Since the morphologies of muscular bundles varied with depth as shown in Fig. 4, the spatially resolved radius was used to characterize their morphologies at different depths. The regions $(1.4 \mathrm{~mm} \times 2.1 \mathrm{~mm})$ in Figs. 5(a)-5(c) were chosen as ROIs to calculate the spatially resolved radius.

Figure 6(a) demonstrates the mean and median of the spatially resolved radius in the ROIs at different depths of myocardium pulmonary junction. The fluctuation of the median radius is smaller than the mean radius. Figure 6(c) demonstrates that the median value could better quantify the size of the muscular bundle than the mean value [Fig. 6(b)], since the smaller radius of speckle noise brought the averaged radius of all three cases to a similar level. On the other hand, the muscular bundles could be segmented from the en face OCT image in the superficial layer [Fig. 5(a)] and the deeper regions [Fig. 5(c)]. However, there is no significant difference between the superficial layer and the deeper region.

\section{Discussions and Conclusions}

In this work, we investigated the atrial myocardium extension into the PVs and the depth-resolved muscular bundles distribution in myocardium pulmonary junction using OCT. Histology remains the gold standard for investigating the microscopic structures of biological tissues. However, histological assessment is time-consuming and can only image a limited area of the fixed tissue. Although we cannot characterize the orientation of fiber bundles, the cross-sectional OCT binary images and depth-resolved en face OCT images were applied for recognizing the myocardial extensions and analyzing the muscular bundles, respectively. Depth-resolved radius of muscular bundles at the junction was also extracted for quantification of muscular bundles at different depths. The median radius of muscular bundles can be used to distinguish the typical three layers in myocardium pulmonary junction. The fiberbased SS-OCT is easily integrated into a catheter, which can potentially provide guidance information for radio-frequency ablation in AF treatment. The results demonstrate that based on image intensity, the cross-sectional binary images can distinguish the PV, myocardium, and myocardium pulmonary junction. It can also quickly estimate the extensions of the muscular bundle in the PV.

\section{Disclosures}

The authors have no relevant financial interests in this article and no potential conflicts of interest to disclose.

\section{Acknowledgments}

Zhifang $\mathrm{Li}$ acknowledges the support from the National Science Foundation of China (No. 81571726), and Yu Chen acknowledges the support from the National Institutes of Health (Nos. R21EB019161-01A1 and R01EB01494601A1). Part of the work has been previously published in SPIE proceedings: $\mathrm{Z}$. Li et al., "OCT imaging of myocardium extending to pulmonary vein," Proc. SPIE 9689, 96893A (2016).

\section{References}

1. C. T. January et al., "2014 AHA/ACC/HRS guideline for the management of patients with atrial fibrillation: executive summary: a report of the American College of Cardiology/American Heart Association task force on practice guidelines and the Heart Rhythm Society," Circulation 130(23), 2071-2104 (2014).

2. M. Haïssaguerre et al., "Spontaneous initiation of atrial fibrillation by ectopic beats originating in the pulmonary veins," N. Engl. J. Med. 339(10), 659-666 (1998).

3. A. D'silva and M. Wright, "Advances in imaging for atrial fibrillation ablation," Radiol. Res. Pract. 2011(2090-1941), 1-10 (2011).

4. C. P. Fleming et al., "Quantification of cardiac fiber orientation using optical coherence tomography," J. Biomed. Opt. 13(3), 030505 (2008).

5. C. J. Goergen et al., "Optical coherence tractography using intrinsic contrast," Opt. Lett. 37(18), 3882-4388 (2012).

6. C. M. Ambrosi et al., "Quantification of fiber orientation in the canine atrial pacemaker complex using optical coherence tomography," J. Biomed. Opt. 17(7), 071309 (2012).

7. G. Yu and C. P. Fleming, "Extracting three-dimensional orientation and tractography of myofibers using optical coherence tomography," Biomed. Opt. Express 4(10), 2150-2165 (2013).

8. C. Fan and Y. Gang, "Imaging myocardial fiber orientation using polarization sensitive optical coherence tomography," Biomed. Opt. Express 4(3), 460-465 (2013).

9. Y. Wang et al., "Histology validation of mapping depth-resolved cardiac fiber orientation in fresh mouse heart using optical polarization tractography," Biomed. Opt. Express 5(8), 2843-2855 (2014).

10. K. P. Quinn and I. Georgakoudi, "Rapid quantification of pixel-wise fiber orientation data in micrographs," J. Biomed. Opt. 18(4), 046003 (2013).

11. W. Yao et al., "Collagen fiber orientation and dispersion in the upper cervix of non-pregnant and pregnant women," Plos One 11(11), e0166709 (2016).

12. Z. Liu et al., "Rapid three-dimensional quantification of voxel-wise collagen fiber orientation," Biomed. Opt. Express 6(7), 2294-2310 (2015).

13. Q. Li et al., "Automated quantification of microstructural dimensions of the human kidney using optical coherence tomography (OCT)," Opt. Express 17(18), 16000-16016 (2009).

14. Q. Tang et al., "Real-time epidural anesthesia guidance using optical coherence tomography needle probe," Quant. Imaging Med. Surg. 5(1), 118-124 (2015).

15. Z. Ding et al., "Imaging spinal structures with polarization-sensitive optical coherence tomography," IEEE Photonics J. 8(5), 6804508 (2016).

16. Q. Tang et al., "Depth-resolved imaging of colon tumor using optical coherence tomography and fluorescence laminar optical tomography," Biomed. Opt. Express 7(12), 5218-5232 (2016).

17. B. Wang et al., "Optical coherence tomography and computer-aided diagnosis of a murine model of chronic kidney disease," J. Biomed. Opt. 22(12), 1-11 (2017).

18. N. Otsu, "Threshold selection method from gray-level histograms," IEEE Trans. Syst. Man Cybern. 9(1), 62-66 (1979).

19. M. Fathy and M. Y. Siyal, "An image detection technique based on morphological edge detection and background differencing for real-time traffic analysis," Pattern Recognit. Lett. 16, 1321-1330 (1995).

20. L. Lam, S. W. Lee, and C. Y. Suen, "Thinning methodologies," IEEE Trans. Pattern Anal. Mach. Intell. 14(9), 869-885 (1992).

21. W. F. Cheong, S. A. Prahl, and A. J. Welch, "A review of the optical properties of biological tissues," IEEE J. Quantum Electron. 26(12), 2166-2185 (1990).

22. D. Levitz et al., "Determination of optical scattering properties of highly-scattering media in optical coherence tomography images," Opt. Express 12(2), 249-259 (2004)

Biographies for the authors are not available. 